\title{
FOETAL AND POSTNATAL EQUINE ARTICULAR CARTILAGE DEVELOPMENT: MAGNETIC RESONANCE IMAGING AND POLARISED LIGHT MICROSCOPY
}

\author{
Caroline Cluzel $^{1,2}$, Laurent Blond ${ }^{2}$, Pascal Fontaine ${ }^{1,2}$, Julien Olive ${ }^{2}$ and Sheila Laverty ${ }^{1,2, *}$ \\ ${ }^{1}$ Comparative Orthopaedic Research Laboratory, Faculty of Veterinary Medicine, University of Montreal, \\ Québec, Canada \\ ${ }^{2}$ Department of Clinical Sciences, Faculty of Veterinary Medicine, University of Montreal, Québec, Canada
}

\begin{abstract}
Adult articular cartilage (AC) has a well described multizonal collagen structure. Knowledge of foetal AC organisation and development may provide a prototype for cartilage repair strategies, and improve understanding of structural changes in developmental diseases such as osteochondrosis (OC). The objective of this study was to describe normal development of the spatial architecture of the collagen network of equine $\mathrm{AC}$ using $1.5 \mathrm{~T}$ magnetic resonance imaging (MRI) and polarised light microscopy (PLM), at sites employed for cartilage repair studies or susceptible to OC. T2-weighted fast-spin echo (FSE) sequences and PLM assessment were performed on distal femoral epiphyses of equine foetuses, foals and adults. Both MRI and PLM revealed an early progressive collagen network zonal organisation of the femoral epiphyses, beginning at 4 months of gestation. PLM revealed that the collagen network of equine foetal $\mathrm{AC}$ prior to birth was already organised into an evident anisotropic layered structure that included the appearance of a dense tangential zone in the superficial AC in the youngest specimens, with the progressive development of an underlying transitional zone. A third, increasingly birefringent, radial layer developed in the AC from 6 months of gestation. Four laminae were observed on the MR images in the last third of gestation. These included not only the AC but also the superficial growth plate of the epiphysis. These findings provide novel data on normal equine foetal cartilage collagen development, and may serve as a template for cartilage repair studies in this species or a model for developmental studies of OC.
\end{abstract}

Keywords: Horse; foetus; histology; magnetic resonance imaging; epiphyseal cartilage; collagen; osteochondrosis; cartilage.

*Address for correspondence:

Sheila Laverty, MVB, Dipl. ACVS, ECVS

Comparative Orthopaedic Research Laboratory

Department of Clinical Sciences

Faculty of Veterinary Medicine

University of Montreal

3200, rue Sicotte

C. P. 5000

Saint-Hyacinthe

Quebec J2S 7C6

Canada

Telephone Number : 1-450-773-8521, extension 8267

FAX Number : 1-450-778-8102

E-mail : sheila.laverty@umontreal.ca

\section{Introduction}

Type II collagen is one of the most abundant ( $80 \%$ dry weight) articular cartilage (AC) matrix molecules and its highly organised network imparts mechanical properties of tensile strength and resistance to shear forces. Adult AC has a zonal or layered structure, created by the predominant collagen fibre orientation, revealed by birefringence on polarised light microscopy (PLM) examination (Benninghoff, 1925). The collagen fibres' preferential direction is either parallel to the articular surface (tangential), lack a preferential direction (transitional), or aligned perpendicular (radial) to the subchondral bone to create the "Benninghoff structure". Furthermore, the number and thickness of collagen fibrils vary with zone (Aspden and Hukins, 1981; Changoor et al., 2011; Hwang et al., 1992), and the structure undergoes major changes with maturation from juvenile to adult cartilage. These changes include an increase in type II collagen content and cross-links and a reorientation of the predominant collagen fibre direction from a parallel to perpendicular orientation to the articular surface (Julkunen et al., 2009; Williamson et al., 2003). Numerous studies describe the biochemical and structural adaptations during postnatal growth and maturation due to postnatal mechanical forces (Brama et al., 2002; Brama et al., 2000; Hughes et al., 2005; Hunziker et al., 2007; Julkunen et al., 2009; Lecocq et al., 2008; van Turnhout et al., 2011), and the functional properties change as the collagen content and collagen fibril orientation mature (Rieppo et al., 2009).

However, AC structure is reported to be homogeneous or isotropic at birth, based on its cellular and histologic appearance, and becomes anisotropic, developing stratified layers with maturity (Hunziker et al., 2007). Cartilage mechanical properties depend on the collagen ultrastructure, and are acquired during collagen network maturation (Julkunen et al., 2009; Julkunen et al., 2008). Although horses are reported to be born with biochemically "blank" (Brama et al. 2002) or uniform joints in respect to collagen content (Brama et al., 2000), this form of assessment does not reveal the spatial architecture of the collagen network. It is proposed that the collagen network at birth is not arranged in a zonal or laminar way as in adult animals (Brama et al., 2002; Holopainen et al., 2008; Hunziker et al., 2007; Julkunen et al., 2009; Rieppo et al., 2009; van Turnhout et al., 2010). The predominant collagen orientation as assessed by quantitative PLM is reported to be parallel to the articular surface, and with age it changes to the classic Benninghoff structure (Julkunen et al., 2009; Julkunen et al., 2010; Rieppo et al., 2009; van Turnhout et al., 2008; van Turnhout et al., 2010). However, there have been 
few studies addressing the in utero collagen structure of AC. Investigations combining PLM and picrosirius red staining for collagen fibres recently provided insight into the collagen network structure of equine foetal $\mathrm{AC}$ and its development: they suggest that it possesses an anisotropic organisation in utero (Lecocq et al., 2008). A multizonal collagen structure is apparent and birefringence increases with gestational age, indicating an increase in collagen content or alignment (Lecocq et al., 2008). Consistent with this observation, a zone with a weak anisotropic collagen arrangement is detectable in perinatal animals employing quantitative PLM (van Turnhout et al., 2010).

Magnetic resonance imaging (MRI) is presently the most accurate imaging modality for evaluating AC in vivo and is now also employed to assess cartilage repair strategies (Hayter and Potter, 2011; Kangarlu and Gahunia, 2006; Uhl et al., 2005). Its principal advantage is that it allows a non-invasive, non-destructive assessment and, unlike many histological techniques, in multiple planes. There is no need for special preparation of the specimen, and the same specimen can be imaged at different times with different techniques, which may save time and preserve the specimen.

Hyaline cartilage has a visible laminar structure (layers of different intensity) on high-resolution magnetic resonance (MR) images that correlates with its histological zonal organisation (Recht et al., 2005). MRI T2 relaxation times reflect the collagen fibril network's predominant structure (Goodwin and Dunn, 1998; Grunder et al., 1998; Mosher and Dardzinski, 2004; Nieminen et al., 2001; Nissi et al., 2006; Xia et al., 2001), the collagen content (Menezes et al., 2004; Mosher and Dardzinski, 2004) and also collagen-associated water (Mosher and Dardzinski, 2004; Nissi et al., 2006). The laminar appearance on T2weighted images reflects the collagen orientation in the superficial zone (fibres parallel to the articular surface), transitional zone (random arrangement) and deep or radial zone (perpendicular to bone surface) (Xia et al., 2001). Consequently, MRI is a valuable tool for assessing the cartilage collagen network non-destructively.

Changes in the collagen network are observed on T2weighted images of cartilage in young postnatal animals: cartilage of very young animals displays a multi-laminar appearance, up to 7 layers (Nissi et al., 2006), whereas a trilaminar adult structure appears with maturity (Grunder et al., 2000; Grunder et al., 1998; Nieminen et al., 2000; Nissi et al., 2006; Xia, 2003). Nissi et al. (2006) conclude that the appearance is due to incomplete maturation and remodelling of the collagenous network with maturity and that up to $44 \%$ of the depth-wise variation in T2 is created by collagen anisotropy. T2-weighted 1.5T MR images reveal signal variations with age in bovine post-natal cartilage concomitant with modifications in its structure (Olivier et al., 2001). However, with routine clinical MRI (1.5T), the multi-laminar appearance is not visible in adult AC (Link et al., 2007; Verstraete et al., 2004).

MRI has rarely been employed to assess foetal AC structure. Connolly et al. (2004) describe prenatal skeletal development features of the epiphysis in pigs that were confirmed on histology. Prenatal MRI is also now emerging clinically as a method to assess skeletal dysplasias, such as collagenopathy mutation of the COL2A1 gene (type II collagenopathy) (Yazici et al., 2010).

Knowledge of normal AC structure during various stages of development is important towards understanding and enhancement of the maturation of cartilage repair techniques in vivo to engineer functional durable cartilage. The creation of an adult hyaline cartilage with a stratified collagen architecture is an important goal for a mechanically functional tissue (Responte et al., 2007; Riesle et al., 1998). Strategies for repairing focal AC defects include surgical microfracture, cell therapy and tissue-engineered constructs (Farr et al., 2011). Healing of these focal defects will probably recapitulate some, or all, of AC matrix developmental events in vivo, as they mature in situ. Similarities between in vitro tissue engineered collagen network structure and that of young calves has been previously identified using scanning electron microscopy (Riesle et al., 1998), but stratification is not addressed. So far stratification of AC repair sites is not reported in animal models on follow up MRI, even when high field MRI (Kim et al., 2010; Watanabe et al., 2009) is employed, and a corresponding disorganised collagen structure is confirmed on PLM examination (Watanabe et al., 2009).

The horse is now recognised as being an appropriate animal in which to test cartilage repair strategies, because the thickness of the $\mathrm{AC}$ of the distal femur is similar to that of humans (Frisbie and Stewart, 2011). Consequently, understanding the AC structure during various stages of development is important in this species. Furthermore, knowledge of the normal collagen network structure in the foetus and foal will provide an important foundation to study developmental diseases of articular cartilage, such as osteochondrosis (OC). This developmental problem arises in the early weeks post-partum (Carlson et al., 1995; Dik et al., 1999; Olstad et al., 2007) and potentially in the foetus (Carlsten et al., 1993; Ekman et al., 2009), and altered collagen metabolism is proposed as a potential contributing factor to this disease (Billinghurst et al., 2004; Bridges and Harris, 1988; Hurtig et al., 1993; Laverty et al., 2000; Laverty et al., 2002; van de Lest et al., 2004).

We hypothesised that $1.5 \mathrm{~T} \mathrm{MRI}$ and picrosirius red histological staining would reveal progressive maturation of equine AC structure in utero. Specifically, we studied the maturation of the distal femoral epiphyseal articular surface, employing both MRI and PLM with picrosirius red staining, at different sites. The aims of our study were to characterise the MRI appearance of the developing equine articular surface and the histological events in the establishment of the collagen network of developing AC.

\section{Materials and Methods}

\section{Study design}

Equine foetuses ( $n=14$, age range 4.1 to 9.4 months of gestation) from an abattoir and full-term foals or adults ( $n=10$, age range from 1.5 days to 2 years) euthanised at the veterinary teaching hospital were collected between 2008 and 2010. The age of the foetuses was estimated by crown-rump measurement (Bergin et al., 1967). Some of 
Table 1. Magnetic resonance imaging sequences employed to image the developing equine femoral epiphysis.

\begin{tabular}{|c|c|c|}
\hline Sequence & Sagittal T2W FS FSE & Dorsal Dual PD+T2W \\
\hline Mode & $2 \mathrm{D}$ & $2 \mathrm{D}$ \\
\hline FE & 512 & 512 \\
\hline $\mathrm{PE}$ & 256 & 256 \\
\hline $\mathrm{TE}(\mathrm{ms})$ & 105.0 & PD: 24.0; T2: 120.0 \\
\hline TR (ms) & 4000.0 & 4500.0 \\
\hline NEX & 3.00 & 3.00 \\
\hline FOV $(\mathrm{cm})$ & 16.0 & 18.0 \\
\hline Number of slices (Min-Max) & $16-64$ & $6-57$ \\
\hline Slice thickness $(\mathrm{mm})$ & 2.0 & 3.0 \\
\hline Slice spacing $(\mathrm{mm})$ & 0.0 & 0.0 \\
\hline Acquisition time (Min-Max) & 6'40”-34'41" & 7'17'-39'09' \\
\hline Physical resolution XxYxZ & $0.31 \times 0.63 \times 2.00 \mathrm{~mm}$ & $0.35 \times 0.70 \times 3.00 \mathrm{~mm}$ \\
\hline ZIP & 1024 & 512 \\
\hline
\end{tabular}

FE, Frequency encoding; FOV, Field of view; FS, Fat Saturation; FSE, Fast Spin Echo; NEX, Number of excitations; PE, Phase encoding; PD, Proton density; TE, Echo time; TR, Repetition time; ZIP, Zero filling interpretation.
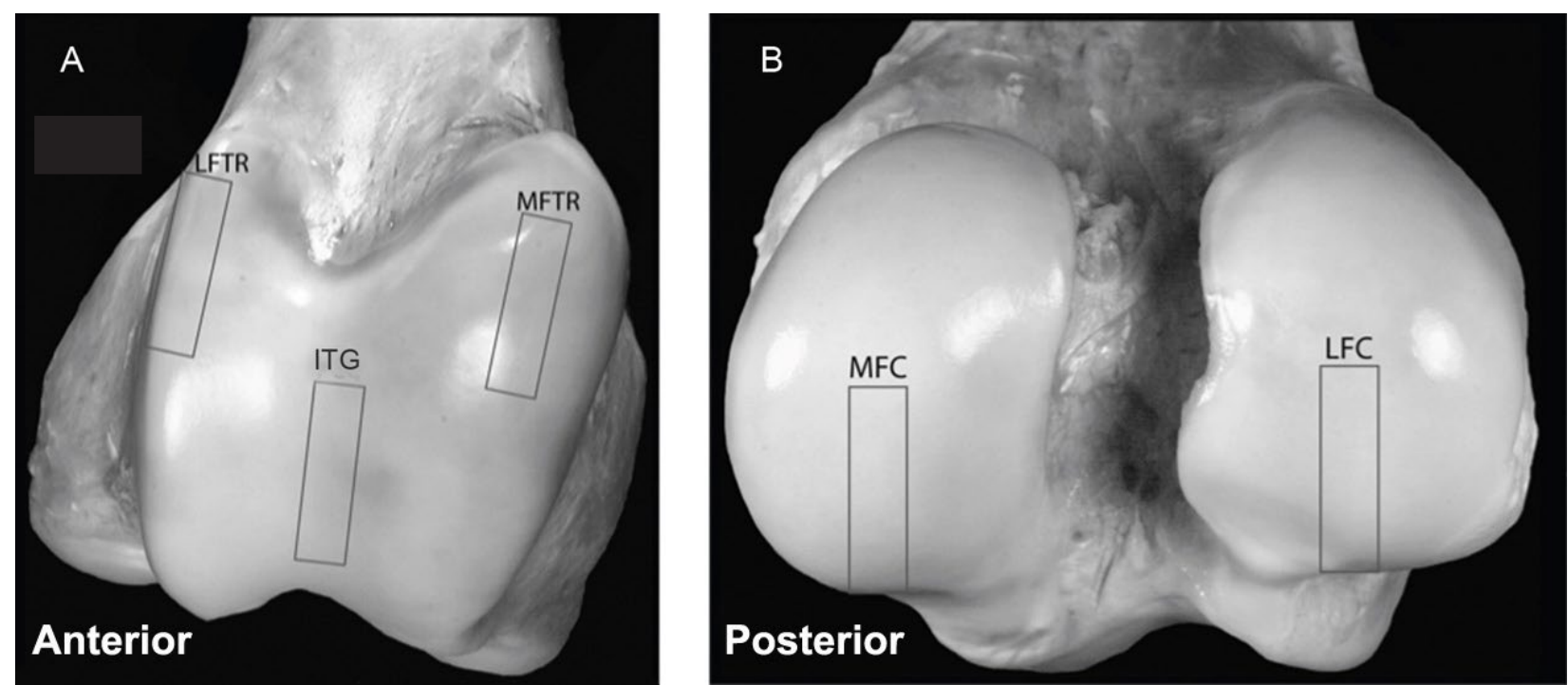

Fig. 1. Distal femoral epiphyseal sites assessed by MRI and PLM. A. Dorsal view of the femoral epiphysis revealing both lateral and medial femoral trochleas and the intertrochlear groove. B. Plantar view of the femoral epiphysis revealing the lateral and medial femoral condyles. The specific sites of interest are outlined by a rectangle. ITG, inter-trochlear groove; LFC, lateral femoral condyle; LFTR, lateral femoral trochlear ridge; MFC, medial femoral condyle; MFTR, medial femoral trochlear ridge.

these specimens were also included in a parallel study of equine joint development (Fontaine et al., 2013). Mature adult specimens were included for comparative purposes with the foetal samples, as MRI features of mature articular cartilage structure are already well described in the literature. Right hind limbs were harvested within $1 \mathrm{~h}$ of death, disarticulated at the coxofemoral joint and were refrigerated at $4{ }^{\circ} \mathrm{C}$ or frozen at $-20{ }^{\circ} \mathrm{C}$ until imaging.

\section{MRI}

Specimens were thawed at room temperature. The distal femurs were then imaged with a 1.5 T magnet MRI (GE Sigma HDX, GE Healthcare Canada, Mississauga, Ontario) employing an 8-channel human knee antenna sent-and- receive coil (HD TRknee PA, GE Healthcare Canada, Mississauga, Ontario). The limb was oriented parallel to the main magnetic field. Two sequences were designed to obtain optimal differentiation of the signal zones in the distal femoral epiphysis: sagittal T2-weighted fastspin echo (FSE) and dorsal dual proton density (PD) + T2-weighted (Table 1). MRI sequence choice was based on those published in prior studies assessing epiphyseal development in various species in the literature (Connolly et al., 2004; Dardzinski et al., 1997; Dardzinski et al. 2002,; Jaramillo et al., 1998; Jaramillo et al., 2004; Varich et al., 2000) and also pilot studies of post mortem specimens to optimise the sequences. If AC lesions were observed, the specimen was excluded from the study. 
Table 2. MRI scores corresponding to atlas of scores in Fig. 4.

\begin{tabular}{|c|c|c|c|c|}
\hline Score & Ossification centre & Layer & Description & See Fig. 4 \\
\hline \multirow{2}{*}{1} & \multirow{2}{*}{ Absent } & 1 & Superficial thin hyperintense & \multirow{2}{*}{ 1B, 1D } \\
\hline & & 2 & Thick isointense (the rest of the epiphysis) & \\
\hline \multirow{3}{*}{2} & \multirow{3}{*}{$<1 / 2$ epiphysis $*$} & 1 & Superficial thin hyperintense & \multirow{3}{*}{$2 \mathrm{~B}, 2 \mathrm{D}$} \\
\hline & & 2 & Thick isointense & \\
\hline & & $3 *$ & Thin hyperintense (surrounding the ossification centre) & \\
\hline \multirow{4}{*}{3} & \multirow{4}{*}{$=1 / 2$ epiphysis } & 1 & Superficial thin hyperintense & \multirow{4}{*}{$3 \mathrm{~B}, 3 \mathrm{D}$} \\
\hline & & 2 & Medium hypointense & \\
\hline & & 3 & Medium isointense & \\
\hline & & 4 & Thin hyperintense (surrounding the ossification centre) & \\
\hline \multirow{3}{*}{4} & \multirow{3}{*}{$>1 / 2$ epiphysis } & 1 & Superficial thin hyperintense & \multirow{3}{*}{$4 \mathrm{~B}, 4 \mathrm{D}$} \\
\hline & & 2 & Medium strongly hypointense & \\
\hline & & 3 & Thin hyperintense (surrounding the ossification centre) & \\
\hline 5 & $100 \%$ epiphysis & 1 & Superficial thin hyperintense & $5 \mathrm{~B}, 5 \mathrm{D}$ \\
\hline
\end{tabular}

* Due to its small size in score 2, the ossification is present inconsistently throughout the epiphysis; when visible on the sagittal view studied, layer 3 is also visible.

\section{MRI analysis}

All digital images were read with a dedicated software (Impax 6.0, Agfa, Toronto, Canada). All sagittal images were assessed and scored (described in detail later). The dorsal images served to locate the five pre-determined sites (Lateral Femoral Trochlear Ridge (LFTR), Medial Femoral Trochlear Ridge (MFTR), Inter-Trochlear Groove (ITG), Lateral Femoral Condyle (LFC), Medial Femoral Condyle (MFC)) for comparative histological analyses (Fig. 1). These specific sites were examined because of their clinical relevance (sites employed for $\mathrm{AC}$ repair strategies or susceptible to OC).

\section{MRI descriptive semiquantitative analysis}

All sagittal images were initially read by the first author and a board-certified veterinary radiologist to form a consensus agreement on the global structural appearance of the epiphysis for each site studied. An atlas was then designed for cartilage pattern score on MRI at different stages of development (foetus to adult). The atlas of scores was made by consensus agreement on the global structural appearance of the epiphysis on T2 sag FSE images in the sagittal plane for each site studied. The scoring system (1-5) incorporated the predominant patterns observed at different stages of development in the sagittal images. Details of the scoring system are provided in Table 2. All images were subsequently placed in random order for interpretation. Each image was scored separately and blindly by the two previous readers and an imaging resident who had never seen the images before, employing the scoring system in Table 2.

\section{MRI quantitative analysis}

The thickness $(\mathrm{mm})$ of the cartilage was measured in a tangential direction from the articular surface using a digital calliper (Fig. 2), and included the whole cartilage depth (both articular and underlying epiphyseal growth cartilage) at all five sites. The number of layers in the cartilage that were clearly visible on MR images was also noted and the thickness $(\mathrm{mm})$ of the superficial hyperintense layer measured. The measurements were made once, by consensus between the author and the board-certified radiologist, at sites corresponding to the site-matched histologic specimens.

\section{Histological analysis}

Following MR imaging, femurs were dissected free of soft tissues. If any macroscopic lesion of the articular surface of the distal femur was observed, the specimen was excluded from the study. The distal epiphysis of the femur was sectioned sagittally with a saw to obtain $3 \mathrm{~mm}$ $x 1.5 \mathrm{~cm}$ sections corresponding to the MR sagittal images at the five sites. The sections were immediately fixed in $10 \%$ formalin for $24 \mathrm{~h}$. They were rinsed in distilled water, before being decalcified in a $1 \mathrm{~L}$ solution containing $170 \mathrm{~mL}$ $12.8 \%$ formic acid, sodium formate $(35 \mathrm{~g})$ and water. If decalcification required more than $48 \mathrm{~h}$ (adult samples), the formic acid solution was replaced every $48 \mathrm{~h}$. After rinsing for $2 \mathrm{~h}$ in running distilled water, the sections were fixed in $70 \%$ alcohol for $48 \mathrm{~h}$. Finally, samples were further dehydrated prior to embedding in paraffin.

Two $4 \mu \mathrm{m}$ thick sections were cut from each block and stained with picrosirius red to reveal collagen structure. The method was adapted from a technique described previously (Sweat et al., 1964), with the inclusion of an additional papain digestion step (K. Pritzker, personal communication; Lecocq et al., 2008). Sections were dewaxed with toluene, washed for $30 \mathrm{~min}$ with water followed by phosphate buffer $(0.05 \mathrm{M}, \mathrm{pH} 4.7)$. They were incubated at $37{ }^{\circ} \mathrm{C}$ in a $0.5 \%$ pre-warmed papain solution (pH 4.4) for 90 min to remove proteoglycans and allow better staining of the collagen fibres with picrosirius red. Slides were washed with distilled water and then placed in $0.1 \%$ picrosirius red solution $(0.1 \mathrm{~g}$ Sirius red F3B, $0.6 \mathrm{~g}$ picric acid crystals, $200 \mathrm{~mL}$ aqueous saturated picric acid) for $60 \mathrm{~min}$. They were then washed in $0.01 \mathrm{M} \mathrm{HCl}$ for $2 \mathrm{~min}$. Finally, they were dehydrated and mounted. Collagen birefringence was then imaged using PLM employing a Leica DM4000B microscope attached to a Leica DFC320 video camera. Highly ordered collagen parallel to the articular surface appears bright and yellow to yellowish orange (birefringent), whereas non-ordered 


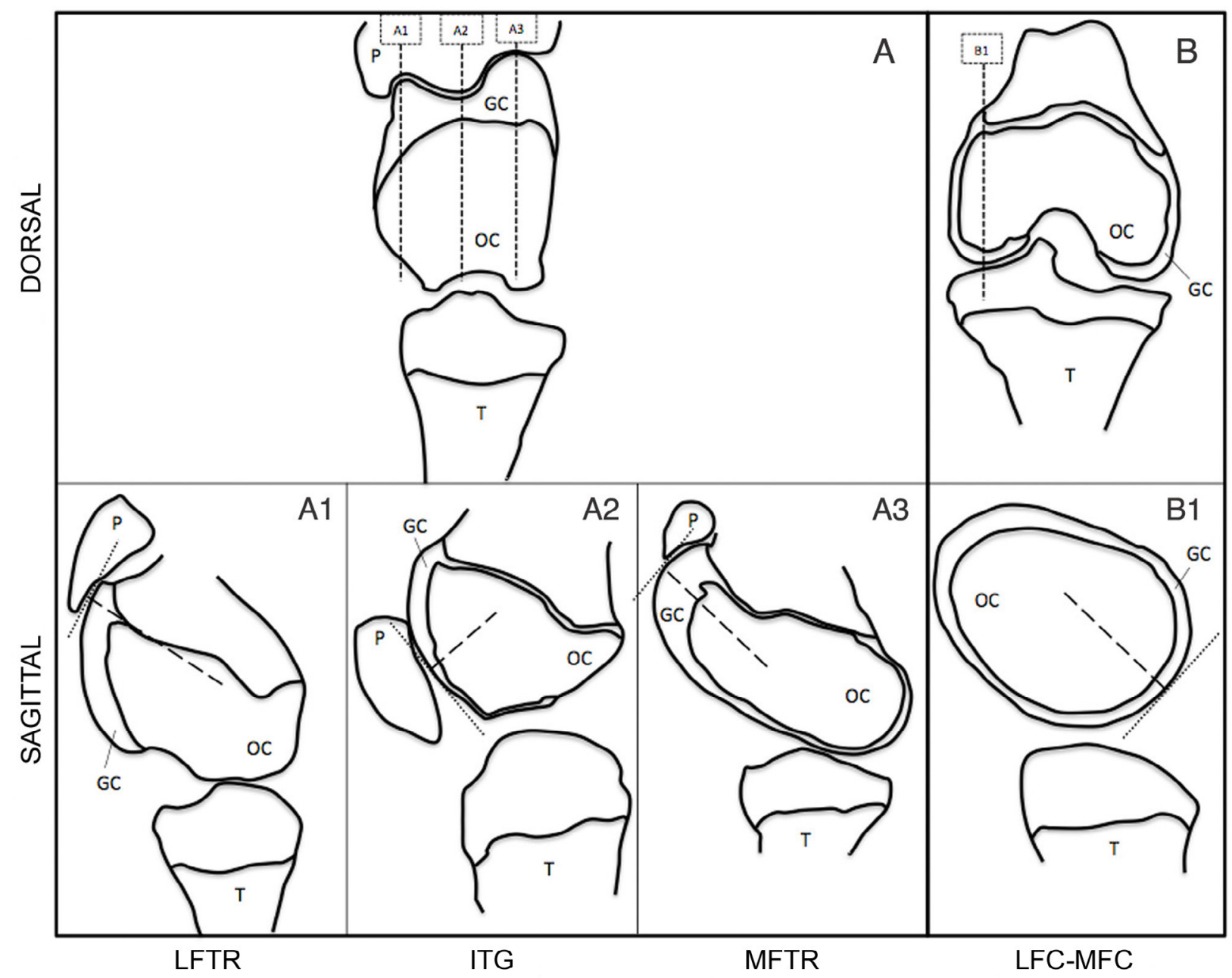

Fig. 2. Cartilage assessment on MRI: thickness measurement. The measurements were made on sagittal sections of the distal epiphysis of the femur. A. Dorsal section of the distal epiphysis of the femur (trochlea) revealing the section axis of the LFTR (A1), ITG (A2) and MFTR (A3) sites. A1-3 illustrate sites of measurement in the sagittal plane on these MRI sections. The thickness of the cartilage $(\mathrm{mm})$ was measured in a tangential direction from the articular surface on the dotted line axis using a digital calliper (precision : $\pm 0.1 \mathrm{~mm}$ ) and included the whole cartilage depth (both articular and underlying epiphyseal growth cartilage). The number of layers in the cartilage that were clearly visible on MRI images was also noted and the thickness of the most superficial layer measured (mm). B. Dorsal section of the distal epiphysis of the femur (condyle) (B is more caudal than A). The dotted line represents the section axis of the LFC (B1). The thickness was measured as in A. GC, growth cartilage; OC, ossification centre of the distal femur; P, patella; T, tibia; ITG, inter-trochlear groove; LFC, lateral femoral condyle; LFTR, lateral femoral trochlear ridge; MFC, medial femoral condyle; MFTR, medial femoral trochlear ridge.

collagen (non-birefringent) appears dark (Hughes et al., 2005; Lecocq et al., 2008). Examination of the collagen structure on the whole slide was performed at low (x12.5, x50) and high (x100, x200, x400) magnifications. Slides were examined at the angle that revealed maximum birefringence of the articular surface.

\section{Histological descriptive semiquantitative analysis}

An atlas of histological scores was made by the first and last authors by consensus agreement to capture the predominant structural appearance of the collagen fibres in the AC, at each site studied. This scoring system (1-5) was based on the birefringence, the predominant pattern of fibre orientation in each layer and the network structure thickness/packing, where discernible (Table 3). Each slide was then scored separately and blindly by the first author and two independent readers who had never seen the slides before, employing Table 3 . These scores were then matched to the ages of the foetuses/foals.

\section{Histological quantitative analysis - articular cartilage thickness}

The thickness ( $\mathrm{mm}$ ) of the AC was measured and the number of zones was noted at the magnification that permitted the best visualisation. There is not a clear boundary for the deep zone of immature cartilage. AC was considered to be the cartilage at the surface of the epiphysis that contained no blood vessels (Klein et al., 2007). Growth cartilage (GC) was defined as cartilage containing cartilage canals. The measurements were made three times on the slides at the same site, and the mean of the three measurements was used for subsequent analyses (Fig. 3). 
Table 3. Histological scores corresponding to atlas of scores in Fig. 6.

\begin{tabular}{|c|c|c|c|}
\hline Score & Zone & Description & See Fig. 6 \\
\hline \multirow{2}{*}{1} & 1 & $\begin{array}{l}\text { Highly birefringent (yellow) } \\
\text { Fibres tangential } \\
\text { Open lattice/spaces spindle-shaped /tangential }\end{array}$ & $1 \mathrm{~A}$ \\
\hline & 2 & $\begin{array}{l}\text { Poorly birefringent (dark) } \\
\text { Fibres random } \\
\text { Open lattice/spaces round to square }\end{array}$ & 1B \\
\hline \multirow{2}{*}{2} & 1 & $\begin{array}{l}\text { Highly birefringent (predominantly yellow) } \\
\text { Fibres tangential } \\
\text { Open lattice/spaces oval to rectangular and tangential }\end{array}$ & $2 \mathrm{~A}$ \\
\hline & 2 & $\begin{array}{l}\text { Mildly birefringent (greenish yellow) } \\
\text { Fibres radial } \\
\text { Open lattice/spaces rectangular to spindle-shaped and radial }\end{array}$ & $2 \mathrm{~B}$ \\
\hline \multirow{3}{*}{3} & 1 & $\begin{array}{l}\text { Highly birefringent (predominantly yellow) } \\
\text { Fibres tangential } \\
\text { Fairly dense lattice/spindle-shaped lattice spaces/tangential }\end{array}$ & $3 \mathrm{~A}$ \\
\hline & 2 & $\begin{array}{l}\text { Poorly birefringent (dark) } \\
\text { Fibres random } \\
\text { Fairly dense lattice/spaces rectangular (tangential proximal/radial distal) }\end{array}$ & $3 \mathrm{~B}$ \\
\hline & 3 & $\begin{array}{l}\text { Mildly birefringent (yellow to greenish) } \\
\text { Fibres radial } \\
\text { Fairly dense lattice/spaces spindle-shaped and radial }\end{array}$ & $3 \mathrm{C}$ \\
\hline \multirow{4}{*}{4} & 1 & $\begin{array}{l}\text { Highly birefringent (bright yellow) } \\
\text { Fibres tangential } \\
\text { Dense lattice/spaces oval and tangential }\end{array}$ & $4 \mathrm{~A}$ \\
\hline & 2 & $\begin{array}{l}\text { Poorly birefringent (dark) } \\
\text { Fibres random } \\
\text { Dense lattice/spaces small round }\end{array}$ & 4B \\
\hline & 3 & $\begin{array}{l}\text { Highly birefringent (yellow to yellowish orange) } \\
\text { Fibres radial } \\
\text { Dense lattice/spaces small round }\end{array}$ & $4 \mathrm{C}$ \\
\hline & 4 & $\begin{array}{l}\text { Poorly birefringent (mainly dark except around chondrocytes:yellow) } \\
\text { Fibres radial } \\
\text { Fairly dense lattice/spaces small round to square }\end{array}$ & $4 \mathrm{D}$ \\
\hline
\end{tabular}

\section{Statistical analysis}

Inter-observer agreement for MRI ( $n=3$ readers) and histological ( $n=3$ readers) scores was calculated using a weighted-kappa coefficient and all sites in all specimens were included. The different sites were also compared to each other with respect to each technique and score, to determine if there was a difference in the measured structures between sites.

The agreement between the MRI scale and the histological scale was assessed using a weightedkappa, globally and for each site separately. An intraclass correlation coefficient was used to determine the association between the thickness of the MRI superficial hyperintense layer and the thickness of AC on histology. The level of significance was set at $P<0.05$. The guidelines for the strength of agreement indicated by the $\kappa$ values were poor $(<0.20)$, fair $(0.21$ to 0.40$)$, moderate $(0.41$ to $0.60)$, substantial ( 0.61 to 0.80$)$, or excellent ( 0.81 to 0.99$)$ (Landis and Koch, 1977).

\section{Results}

Two specimens (6 months and 1 year) were excluded following MRI analysis due to the presence of OC lesions on the LFTR, and one (aged 4.5 years) was excluded due

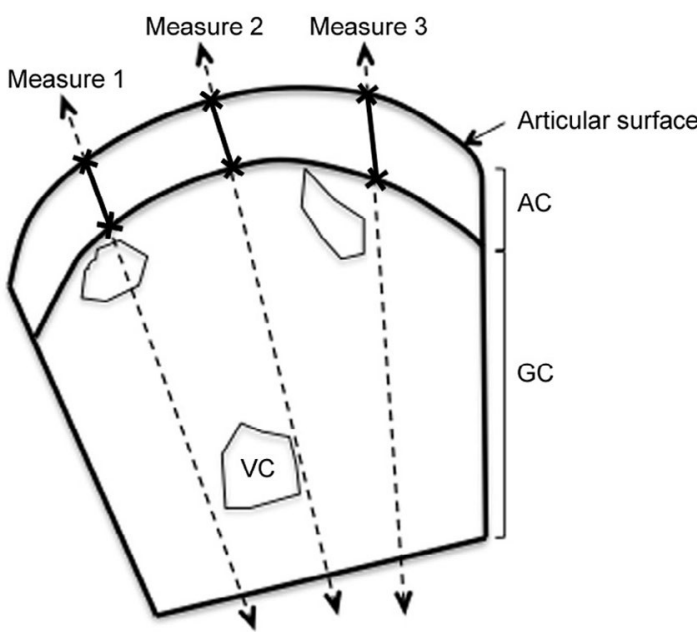

Fig. 3. Articular cartilage measurement on histological sections. The thickness of the articular cartilage $(\mathrm{mm})$ was measured at the magnification that permitted the best visualisation. AC was considered to be the cartilage at the surface of the epiphysis that contained no blood vessels. GC was defined as cartilage housing cartilage canals. The articular surface was divided into quarters. The measurements were made at three locations (full line distance between " $x$ ") on the slides at the same site and the mean of the three measurements was used for subsequent analyses. AC, articular cartilage; GC, growth cartilage; $\mathrm{VC}$, vascular canal. 
to extensive AC surface defects and fibrillation affecting the studied sites. The final samples comprised 14 foetuses, 5 foals and 2 adults for comparative purposes (Table 4).

\section{MRI analysis}

MRI descriptive semiquantitative analysis

There was an excellent inter-observer agreement for MRI scoring between the three readers $(\kappa=0.98,0.93,0.95)$, all sites included.

In the very young foetuses ( $<7-8$ months of gestation), the distal epiphysis of the femur was fully-cartilaginous. The cartilage was composed of the AC on the surface and the deeper GC. MRI revealed a homogeneous isointense tissue throughout the epiphysis with a superficial thin hyperintense layer, that we postulated to be a maturing articular surface, appearing in some of the youngest samples from 4.1 months of gestation (Fig. 4, 1A-D).

The ossification centre of the femoral epiphysis first appeared between 7 and 8 months of gestation. From about 7.4 to 7.6 months of gestation, the secondary ossification centre had expanded to $50 \%$ of the epiphysis. Three layers of cartilage were discernible on MRI between the articular surface and the border of ossification centre. These included the persistent, previously described, superficial thin hyperintense layer (putative future AC) and two additional layers: a thicker iso- to hypointense and a thin hyperintense area surrounding the ossification centre, revealing structural changes in the GC (Fig. 4, 2A-D).

At a later stage in development, in the last third of gestation, four layers of cartilage were visible on the MRI images from the articular surface to the ossification centre. This consisted of the persisting superficial thin hyperintense layer on the surface and surrounding the ossification centre, but a division of the central layer previously described into two layers (a medium hypointense and a medium isointense) (Fig. 4, 3A-D).

With progression of development, from the last days pre-partum to a few weeks post-partum, the MRI cartilage appearance changed to a trilaminar structure: three layers were again visible consisting of the superficial thin hyperintense (articular surface) zone, a middle hypointense zone and a deep hyperintense area (Fig. 4, 4A-D).

From 1 year old, the epiphysis was fully ossified and had the adult appearance. A single superficial thin hyperintense layer remained on the surface of the AC (Fig. 4, 5A-D).

When the dynamics of epiphyseal cartilage development were compared at different sites, the same patterns were present, but the structural changes differed slightly, over time, at each site.

In summary, a superficial hyperintense area became evident on MRI as early as 4.1 months of gestation and persisted in the adult AC. Immediately underneath this zone, a hypointense zone became evident as early as 7.4 months of gestation and also persisted, highlighting structural maturation of the articular surface in utero.

\section{MRI quantitative assessment}

The superficial layer thickness (hyperintense zone) at the 5 sites was not significantly different in the youngest foetuses (score 1 and 2). From 8 months of gestation, differences
Table 4. Age and sex of the equine specimens.

\begin{tabular}{|c|c|c|}
\hline No. & Age & Sex \\
\hline 1 & $4.1 \mathrm{MOG}$ & $\mathrm{F}$ \\
\hline 2 & $4.6 \mathrm{MOG}$ & $\mathrm{M}$ \\
\hline 3 & $4.6 \mathrm{MOG}$ & $\mathrm{M}$ \\
\hline 4 & $4.7 \mathrm{MOG}$ & $\mathrm{F}$ \\
\hline 5 & $6.0 \mathrm{MOG}$ & $\mathrm{M}$ \\
\hline 6 & $6.6 \mathrm{MOG}$ & $\mathrm{M}$ \\
\hline 7 & $7.1 \mathrm{MOG}$ & $\mathrm{F}$ \\
\hline 8 & $7.4 \mathrm{MOG}$ & $\mathrm{F}$ \\
\hline 9 & $7.6 \mathrm{MOG}$ & $\mathrm{F}$ \\
\hline 10 & $8.0 \mathrm{MOG}$ & $\mathrm{M}$ \\
\hline 11 & $8.2 \mathrm{MOG}$ & $\mathrm{F}$ \\
\hline 12 & $8.5 \mathrm{MOG}$ & $\mathrm{M}$ \\
\hline 13 & $8.7 \mathrm{MOG}$ & $\mathrm{M}$ \\
\hline 14 & $9.4 \mathrm{MOG}$ & $\mathrm{M}$ \\
\hline 15 & $1.5 \mathrm{~d}$ & $\mathrm{~F}$ \\
\hline 16 & $3 \mathrm{~d}$ & $\mathrm{M}$ \\
\hline 17 & $5 \mathrm{~d}$ & $\mathrm{~F}$ \\
\hline 18 & $21 \mathrm{~d}$ & $\mathrm{~F}$ \\
\hline 19 & $52 \mathrm{~d}$ & $\mathrm{M}$ \\
\hline 20 & 1 year & F \\
\hline 21 & 2 years & F \\
\hline
\end{tabular}

F, female; M, male; MOG, months of gestation.

were present. There was a significant difference between the mean superficial layer thickness of the sites studied, in specimens with score $3(P<0.0001), 4(P=0.01)$ and 5 $(P=0.007)$. Tukey's post-hoc test indicated that this layer in LFC and MFC was significantly thinner than it was in the trochlear sites for score 3, ITG was significantly thicker than MFC for score 4, and MFTR was significantly thicker than LFC, MFC and LFTR for score 5 (Fig. 5).

\section{Histologic analysis}

Histologic descriptive semiquantitative analysis

There was a very good inter-observer agreement (three readers) for histological scoring $(\kappa=0.86,0.81,0.82)$.

In the early foetal age (before 6 months of gestation), the collagen network was a very loose meshwork and homogenous (Fig. 6, 1B) showing no birefringence, except for the thin tangential zone at the surface (yellow to orange, but loose network) (Fig. 6, 1A).

At 6 months of gestation, a radial zone (Fig. 6, 2B) appeared under the surface tangential zone (Fig. 6, 2A), but was still very loosely woven. It was mildly birefringent, greenish yellow, with the predominant collagen fibre pattern now oriented radially.

With progression of development, at all sites, a transitional zone (Fig. 6, 3B) became visible (earliest stage at 6.6 months of gestation) between the two pre-existing zones: it was poorly birefringent, quite dark, with a random organisation of the fibres. The whole lattice became fairly dense and the radial zone was now well visible (Fig. 6, 3C).

From the end of gestation to a few weeks post-partum, an additional zone was apparent in the deepest part of the $\mathrm{AC}$ at the junction with the GC: it appeared to correspond to the calcified cartilage (Fig. 6, 4D). 


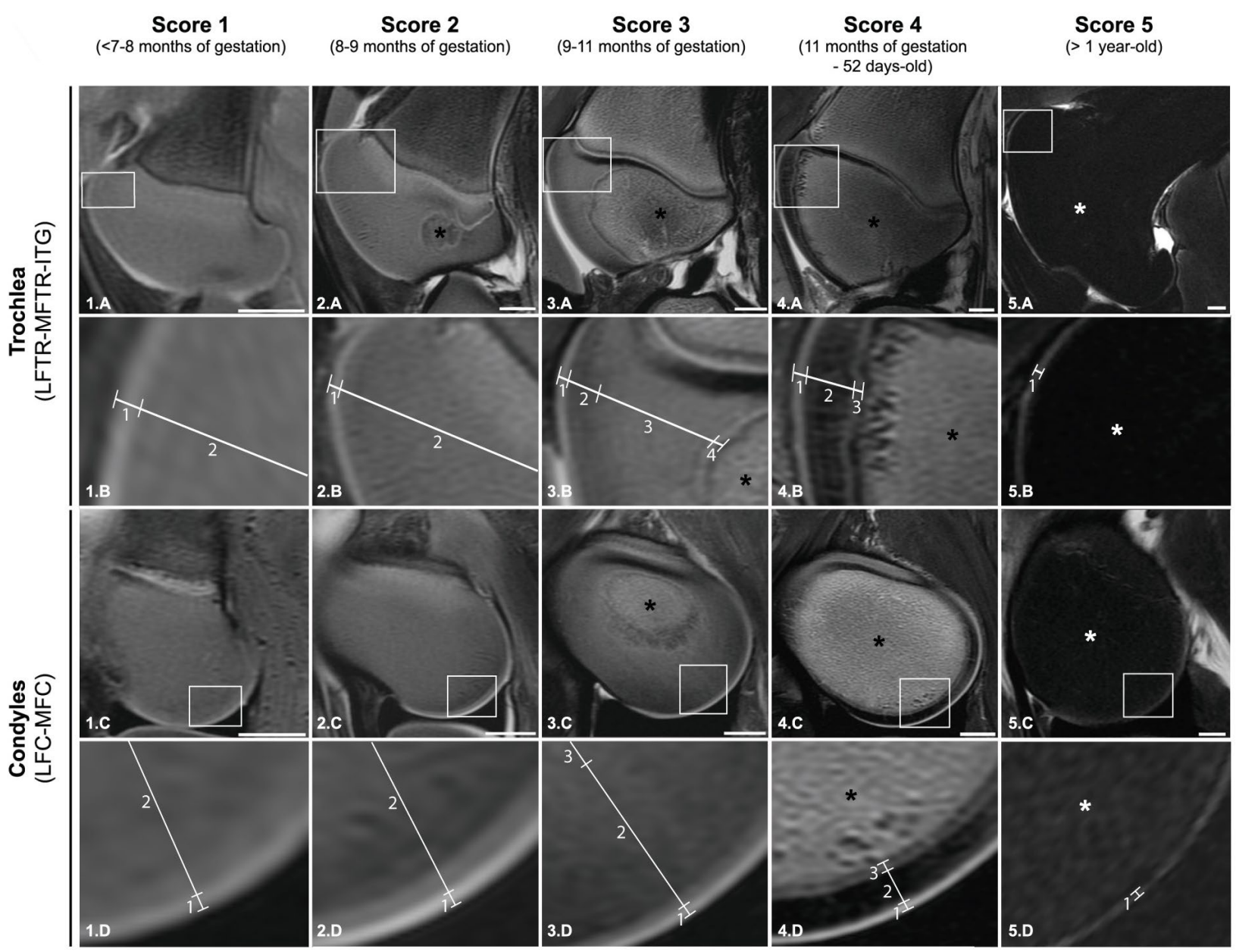

Fig. 4. Atlas designed for cartilage pattern score on MRI at different stages of development (foetus to adult). The atlas of scores was made by consensus agreement of the global structural appearance of the epiphysis on T2 sag FSE images in the sagittal plane for each site studied. It was designed to capture the predominant patterns (ossification centre and cartilage layers) observed at different stages of development. 1.B to 5.B and 1.D to 5.D are magnifications of the sections in rectangles in 1.A to 5.A and 1.C to 5.C, respectively. Layers are delimitated by segments and numbered as in Table 2. 2.B, 2.C and 2.D do not show an ossification centre because of its small size, but it was present inconsistently throughout the T2 sag FSE sequence. The age of each specimen is mentioned: for foetuses, the age is estimated foetal gestational age (see Materials and Methods). * Ossification centre. Scale bars $=10 \mathrm{~mm}$.

The final adult collagen organisation (first present at 1 year-old) had four distinct layers, and a dense lattice that rendered difficult to distinguish the fibre orientation: a superficial yellow to orange tangential zone (Fig. 6, 5A), a dark thin transitional zone (Fig. 6, 5B), a broad yellow radial zone (Fig. 6, 5C), and a poorly birefringent zone in contact with bone (calcified cartilage) (Fig. 6, 5D).

Histological quantitative analysis: articular cartilage thickness

The mean AC thickness at the five sites was not significantly different in the youngest foetuses (score 1 and 2). From 8 months of gestation, differences in thickness were evident. There was a significant difference between the mean AC thickness of the sites studied for score $3(P=0.01)$, score $4(P<0.0001)$ and score $5(P=0.04)$. Tukey's post-hoc tests indicated that the AC of LFC was both significantly thinner than the AC in LFTR and MFTR with score 3,
LFTR and MFTR were significantly thicker than the other sites for score 4, MFC was significantly thicker than ITG for score 4, and ITG was slightly significantly thicker than LFC for score 5 (Fig. 5).

\section{Comparison: MRI vs. histology}

The correlation between the 2 scales of scores was good $(\kappa=0.66)$, all sites included. It was better for LFC and MFTR ( $\kappa=0.69$ and 0.66 , respectively) than MFC and LFTR ( $\kappa=0.63$ and 0.58 , respectively). The kappa was not available for ITG.

The histological thickness of AC correlated with the measurements of the superficial MRI hyperintense layer thickness in $57 \%$ of the measures, all sites and scores included. The MFTR, LFTR and MFC had a better correlation rate $(66.3 \%, 63.2 \%$ and $61.7 \%$, respectively) than the ITG and LFC ( $44.5 \%$ and $42.3 \%$, respectively). 

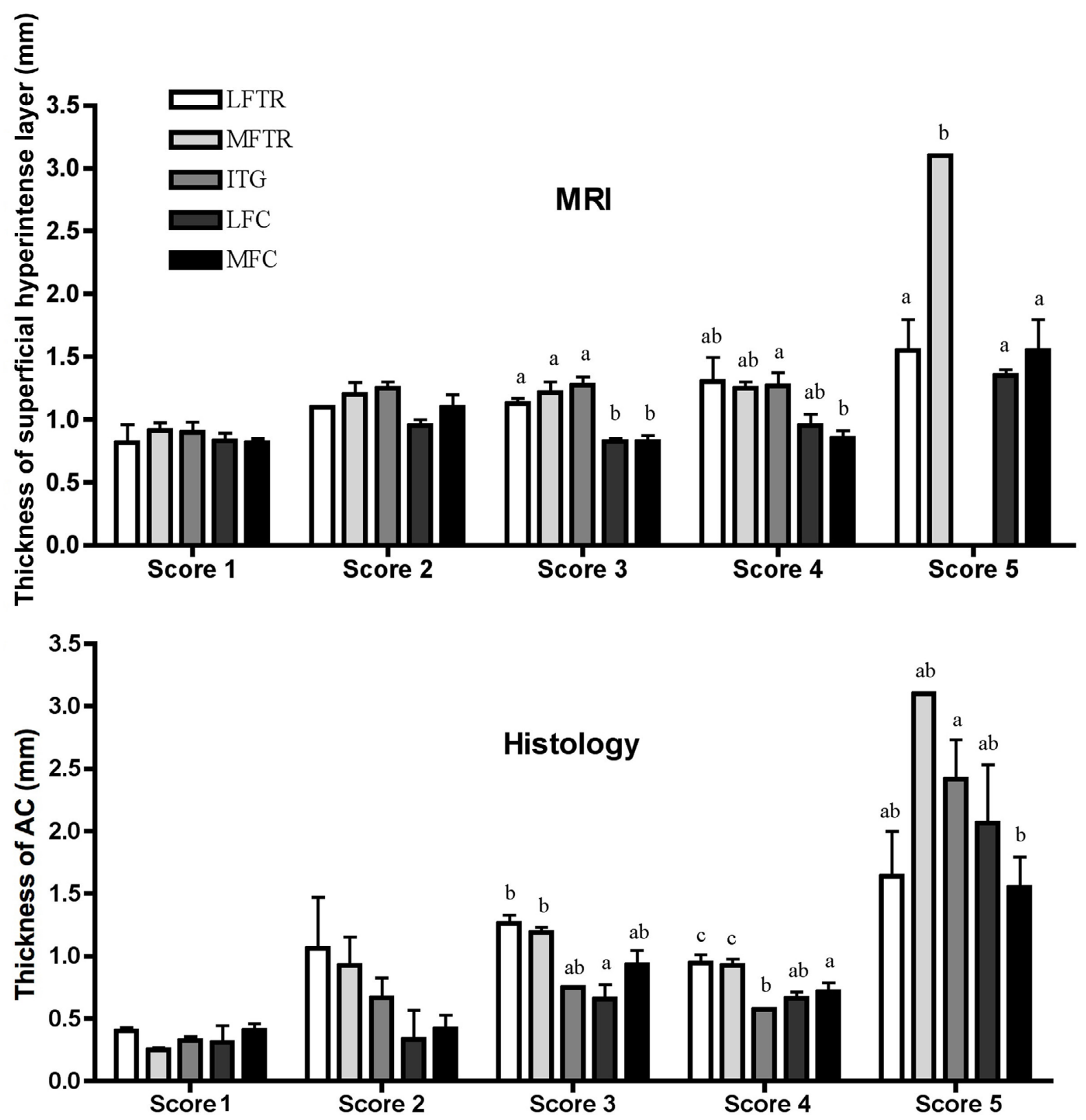

Fig. 5. Thickness of the superficial hyperintense layer on MRI (mm) and thickness of AC on histological slides (mm) for each score and for each site. For each technique, results of Tukey's post-hoc test are indicated with letters (a and b): results that share letters are not significantly different $(P<0.05)$. No measure could have been achieved for ITG on MRI images. ITG, inter-trochlear groove; LFC, lateral femoral condyle; LFTR, lateral femoral trochlear ridge; MFC, medial femoral condyle; MFTR, medial femoral trochlear ridge.

\section{Discussion}

This study provided novel information on the dynamics of the developing collagen network structure in AC in the equine foetus employing both MRI and PLM. This description of normal AC collagen network evolution in the foetus will serve as a benchmark towards understanding desirable collagen network organisation in cartilage engineering strategies in this species and also, potentially, of developmental defects of cartilage such as OC. Both MRI and PLM revealed an early progressive structural laminar/zonal organisation of the collagen network, prior to birth and post-natal load-bearing, though not as pronounced as in adult tissues, due to less collagen content and organisation. A recent study comparing PLM and scanning electron microscopy confirms that collagen fibre orientation observed on PLM reflects cartilage collagen ultrastructure validating this technique for an easy qualitative assessment of the collagen network (Changoor et al., 2011).

As early as 4.1 months gestation age, the earliest time point we assessed, a superficial hyperintense layer was visible at the AC surface on T2-weighted images, which persisted throughout gestation and into adulthood. The mean gestational period in the horse is eleven months. It is now well recognised that $\mathrm{T} 2$-weighted images reflect 


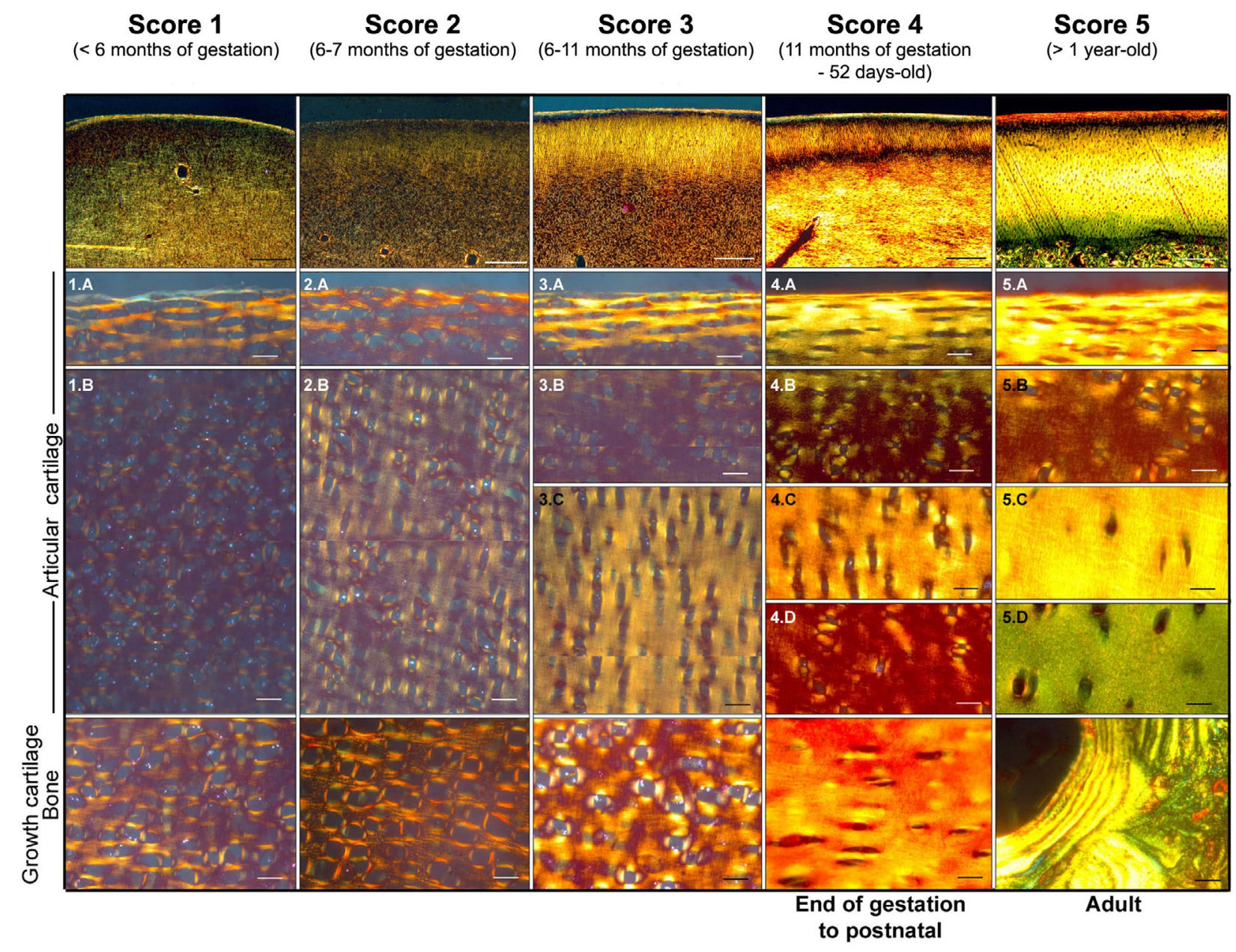

Fig. 6. Histological scores employed for articular cartilage assessment. The atlas of histological scores was made by consensus agreement to capture the predominant structural appearance of the collagen fibres in the articular cartilage on the picrosirius red-stained sections. This scoring system was based on the birefringence, the predominant pattern of fibre orientation in each zone, and the network structure thickness/packing, where discernible. The age of each specimen is mentioned: for foetuses, the age is estimated foetal gestational age (see Materials and Methods). Scale bars $=500 \mu \mathrm{m}$ (first row), $20 \mu \mathrm{m}$ (others).

principally collagen network structure (Grunder et al., 1998; Mosher and Dardzinski, 2004; Nieminen et al., 2001; Nissi et al., 2006; Xia et al., 2001).

A superficial tangential birefringent zone was also present from the earliest stages on PLM, confirming and extending our previous findings (Lecocq et al., 2008). This layer persisted throughout development but became increasingly birefringent. Its dense appearance suggested enhanced collagen deposition and increased collagen content. A thin layer of higher collagen content has also been recently described in human AC foetal specimens (Mahmoodian et al., 2011). The surface birefringent layer we described, is also observed in new-born mice (Hughes et al., 2005) on PLM, but they were not assessed at the foetal stage. Similarly, AC surface anisotropy has been measured on quantitative PLM in stillborn foals, but the overall predominant collagen fibre orientation is parallel to the surface employing these methods (van Turnhout et al., 2008). However, it should be pointed out that the use of orientation patterns alone for determination of the different zones might not suffice for young animals (van Turnhout et al., 2008). As the superficial zone of AC has recently been shown to contain a slowly proliferating stem cell pool, it is not surprising that it is visible from early developmental stages (Dowthwaite et al., 2004; Hunziker et al., 2007). Based on these findings, we can conclude, at least in this species, that the first superficial layer of the classical Benninghoff structure of AC develops in utero and is the first sign of a maturing collagen network. It should be pointed out that when this layer first appears, the rest of the femoral epiphysis is completely cartilaginous, and ossification has not occurred. The cartilage below the superficial intense zone has a homogeneous loose lattice or meshed appearance on PLM.

Four laminae were observed on the MR images in the last third of gestation, but included the complete AC epiphyseal complex with the superficial growth plate of the epiphysis. In contrast to the adult cartilage appearance on MRI, with three zones, up to seven zones of different intensity have already been observed in juvenile femoral cartilage on MRI in various species (Grunder, 2006; Grunder et al., 2000; Grunder et al., 1998; Nieminen et al., 2000; Nissi et al., 2006; Xia et al., 2003). 
Additional evidence for collagen network structure anisotropy in the foetus is provided by the observations of the emergence of a transitional zone with a third increasingly birefringent radial layer on PLM from 6 months of gestation onwards here and in our previous work (Lecocq et al., 2008). Recently, van Turnhout et al. (2008; 2011) also provide evidence for the presence of a transitional zone at birth on quantitative PLM in both the horse and sheep. Based on our structural observations on PLM here and elsewhere (Lecocq et al., 2008), we propose that it arises earlier in utero in the last third of gestation. Additional lines of evidence for foetal cartilage anisotropy are provided by the measurement of a depthdependent stiffness already in foetal cartilage (Klein et al., 2007). Combined, these observations confirm and extend our previous observations (Lecocq et al., 2008) that the collagen network of equine $\mathrm{AC}$ is already organised into an evident layered structure in an equine foetus prior to birth and is anisotropic. This collagen network is subsequently remodelled after birth to attain the adult structure as elegantly shown by others (Brama et al., 2002; Hunziker et al., 2007; Julkunen et al., 2009; Rieppo et al., 2009; van Turnhout et al., 2011) due to growth, maturation as well as joint loading. Briefly, this process involves orchestrated changes that include increased collagen fibre deposition, post-translational collagen fibril cross-linking and maturation of collagen fibre orientation to attain adult collagen mechanical properties.

The signals that orchestrate the development and maturation of the articular surface remain incompletely understood. They include a combination of biomechanical and molecular signals (Onyekwelu et al., 2009). It has been known for some time that skeletal muscle contractions are necessary for normal joint development and that they first occur very early in life then increase in frequency and duration and can occupy up to $80 \%$ of the foetus's time (Murray and Drachman, 1969), as an absence of joint development occurs in chick embryos with continuous application of neuromuscular blocking agents. The involuntary muscle contractions exert traction on the bones and also cause flexions and extensions of the joints creating local tensile strains and shear forces in the cartilage (Carter and Wong, 2003). Ligaments, as they develop and move apart, will also exert forces on the developing joint (Heegaard et al., 1999). Compressive forces arise after birth. Changes in hydrostatic pressure and shear stress within the cartilage influence chondrocyte gene expression and matrix formation and local growth. However, as the forces depend on muscle strength and joint morphology, other factors must be involved to provide the relatively standard homogenous AC appearance and thickness throughout the diarthrodial joints.

Molecular signals also have an important role in joint morphogenesis and work in concert with biomechanical forces to shape the joint. A plethora of growth factors and their receptors also influence AC development in a paracrine fashion (Onyekwelu et al., 2009). It is now believed that a balance between these molecular cues and mechanical stresses contributes to the normal zonal growth of AC. It has been recently shown that molecular signalling alone can induce and enhance $\mathrm{AC}$ maturation in vitro. $\mathrm{A}$ combination of growth factors (FGF-2 and TGF $\beta 1$ ) induce collagen remodelling and significantly enhance (229\% increase) mechanical stiffness and change in the microanatomic structure of cartilage explants from immature calves in vitro without any loading of the construct (Khan et al., 2011). Enhanced crosslinking of the collagen fibrils is also observed. Of importance to the present study, they also observe a response in organisation of the collagen fibrils at the structural level into pericellular, territorial and interterritorial matrices. They note that the differentiation of the extracellular matrix is most apparent at the surface of the explants, similar to our observations with development and maturation of equine foetal cartilage in utero. This important in vitro study indicates that molecular signals alone can potentially guide the structure of the developing collagen network. Taken together, these studies suggest that the zonal structural changes in the collagen network we have observed in utero result from the combined dynamic changes in paracrine growth factors and forces generated by developing muscles and expanding ligaments.

We also identified site differences in AC structure, as reported previously on PLM studies (Lecocq et al., 2008; van Turnhout et al., 2010). The thickness of the AC was also significantly different between the five sites on the distal femur at certain ages, as previously observed by Lecocq et al. (2008). These differences reflect site and joint maturational differences (Lecocq et al., 2008), also observed by others (Brama et al., 2000; Julkunen et al., 2010). Grunder (2006) postulates that the site-related variation of cartilage laminae on MRI is an adaptation of the collagenous network to the regional different "biochemical requirements". Variations in collagen network organisation are also observed in young animals at different sites within the joint (Hyttinen et al., 2009) and also between different articular surfaces (Julkunen et al., 2010).

The horse stands immediately after birth and loads its joints. This may be an example of a species with precocious joint development when compared to mice or rabbits, and it is possible that the foetal AC collagen network may develop differently in different species, because of these different needs, but this remains to be elucidated. The foetal development of the articular cartilage collagen network has not yet been described in any other species, to our knowledge.

The collagen network at AC repair sites, following cell therapy or tissue engineering strategies, may recapitulate similar maturational stages to foetal AC and ideally progressively develop to an organised zonal structure, to meet load requirements. Microfracture is the only technique reported to date, to the best of our knowledge, that produces repair cartilage with a multizonal architecture on PLM: Changoor et al. (2011) observe a stratified organisation of collagen similar to adult cartilage at 1 year in biopsy specimens of the repair site, but there is no information on earlier time points.

There are several limitations to this study. First, we could not access foetal specimens earlier than 4 months gestational age and were not able to determine the earliest apparition of the superficial tangential layer. However, these are the earliest specimens described in the literature 
to our knowledge. Second, MRI technique could have influenced image quality. For example, the main magnetic field, although oriented from distal to proximal relative to the hind limb axis, was not always oriented in the same manner relative to all the articular surfaces examined and could influence the intensity of the layers observed (magic angle effects). It is also important to note that truncation artefact was not considered as a limitation here as it was previously ruled out as the cause of laminae on FSE MR images (Kim et al., 1999). Partial volume effect, underestimation of signal due to small object size, could have lowered the resolution of the MRI images: the choice of constant $3 \mathrm{~mm}$ thick slices has resulted in partial volume effects that were relatively more important in foetal compared with adult femurs. This might have impacted the capacity to identify thin cartilage layers in the smaller subjects. Higher resolution MRI and quantitative MRI sequences such as T2, T1rho mapping or gagCEST imaging (Borthakur and Reddy, 2010) would have provided greater detail and are planned in future studies. In addition, quantitative PLM and scanning electron microscopy would have provided valuable additional information on these specimens.

Joints or limbs are often stored frozen when there is a delay in imaging due to either delay in collection of all specimens or scheduling issues, to attempt to prevent chondrocyte catabolic enzyme degradation of the cartilage matrix (Fishbein et al., 2007). Nevertheless, this is a trade-off as the freeze-thaw process, particularly when repeated, may lead to ice crystal accumulation and potential disruption of the cartilage collagen structure (ap Gwynn et al., 2000; Jomha et al., 2004). On the other hand, investigators have also reported that small ice crystals do not affect the collagen orientation on scanning electron microscopic examination of mice articular cartilage samples (Hughes et al., 2005).

Studies on the effects of freezing on cartilage MRI T2 relaxation have been performed principally on cartilage explants (Fishbein et al., 2007; Reiter et al., 2011) The results of these studies are contradictory but some suggest that freezing may alter collagen structure (Laouar et al., 2007). Freezing and thawing of bovine nasal cartilage explants resulted in small changes in T2 (Fishbein et al., 2007; Reiter et al., 2011). It is not known if these observations of freezing effects on isolated cartilage explants can be extrapolated to complete intact joints.

In conclusion, this study described the MRI appearance of the developing articular surface and histological structure of the surface collagen framework. Knowledge of this primitive collagen network development and organisation may help understanding of the maturation of collagen in cartilage repair sites where developmental events are increasingly recognised to be recapitulated.

\section{Acknowledgements}

We are grateful to Hélène Richard and Suzie Lachance for technical assistance in the PLM and MRI, respectively. The authors thank Guy Beauchamp for statistical data analysis. Furthermore, we would like to thank Raphael Vezina for scoring the histological slides. We wish to confirm that there are no known conflicts of interest associated with this publication and there has been no significant financial support for this work that could have influenced its outcome.

\section{References}

ap Gwynn I, Wade S, Kaab MJ, Owen GR, Richards RG (2000) Freeze-substitution of rabbit tibial articular cartilage reveals that radial zone collagen fibres are tubules. J Microsc 197: 159-172.

Aspden RM, Hukins DW (1981) Collagen organization in articular cartilage, determined by X-ray diffraction, and its relationship to tissue function. Proc R Soc Lond B Biol Sci 212: 299-304.

BenninghoffA (1925) Form und Bau der Gelenkknorpel in ihren Beziehungen zur Funktion [Form and structure of hyaline cartilage in relation to function]. Zeit Zelforsch Mikroscop Anat 2: 783-862.

Bergin WC, Gier HT, Frey RA, Marion GB (1967) Developmental horizons and mesurements useful for age determination of equine embryos and fetuses. Proceedings of the 13th Annual Convention of the American Association of Equine Practitioners, New Orleans, pp 179-196.

Billinghurst RC, Brama PA, van Weeren PR, Knowlton MS, McIlwraith CW (2004) Evaluation of serum concentrations of biomarkers of skeletal metabolism and results of radiography as indicators of severity of osteochondrosis in foals. Am J Vet Res 65: 143-150.

Borthakur A, Reddy R (2010) Imaging cartilage physiology. Top Magn Reson Imaging 21: 291-296.

Brama PA, Tekoppele JM, Bank RA, Barneveld A, van Weeren PR (2000) Functional adaptation of equine articular cartilage: the formation of regional biochemical characteristics up to age one year. Equine Vet J 32: 217 221.

Brama PA, TeKoppele JM, Bank RA, Barneveld A, van Weeren PR (2002) Development of biochemical heterogeneity of articular cartilage: influences of age and exercise. Equine Vet J 34: 265-269.

Bridges CH, Harris ED (1988) Experimentally induced cartilaginous fractures (osteochondritis dissecans) in foals fed low-copper diets. J Am Vet Med Assoc 193: 215-221.

Carlson CS, Cullins LD, Meuten DJ (1995) Osteochondrosis of the articular-epiphyseal cartilage complex in young horses: evidence for a defect in cartilage canal blood supply. Vet Pathol 32: 641-647.

Carlsten J, Sandgren B, Dalin G (1993) Development of osteochondrosis in the talocrural joint and osteochondral fragments in the fetlock joints of Standardbred trotters: I. A radiological survey. Equine Vet J 25 Suppl 16: 42-47.

Carter DR, Wong M (2003) Modelling cartilage mechanobiology. Philos Trans R Soc Lond B Biol Sci 358: 1461-1471.

Changoor A, Nelea M, Methot S, Tran-Khanh N, Chevrier A, Restrepo A, Shive MS, Hoemann CD, Buschmann MD (2011) Structural characteristics of the collagen network in human normal, degraded and repair articular cartilages observed in polarized light and scanning 
electron microscopies. Osteoarthritis Cartilage 19: 14581468.

Connolly SA, Jaramillo D, Hong JK, Shapiro F (2004) Skeletal development in fetal pig specimens: MR imaging of femur with histologic comparison. Radiology 233: 505514.

Dardzinski BJ, Mosher TJ, Li S, Van Slyke MA, Smith MB (1997) Spatial variation of T2 in human articular cartilage. Radiology 205: 546-550.

Dardzinski BJ, Laor T, Schmithorst VJ, Klosterman L, Graham TB (2002) Mapping T2 relaxation time in the pediatric knee: feasibility with a clinical 1.5-T MR imaging system. Radiology 225: 233-239.

Dik KJ, Enzerink E, van Weeren PR(1999) Radiographic development of osteochondral abnormalities, in the hock and stifle of Dutch Warmblood foals, from age 1 to 11 months. (Osteochondrosis and musculoskeletal development in the foal under the influence of exercise). Equine Vet J 31 Suppl 31: 9-15.

Dowthwaite GP, Bishop JC, Redman SN, Khan IM, Rooney P, Evans DJ, Haughton L, Bayram Z, Boyer S, Thomson B, Wolfe MS, Archer CW (2004) The surface of articular cartilage contains a progenitor cell population. J Cell Sci 117: 889-897.

Ekman S, Carlson CS, van Weeren PR (2009) Workshop report. Third International Workshop on Equine Osteochondrosis, Stockholm, 29-30th May 2008. Equine Vet J 41: 504-507.

Farr J, Cole B, Dhawan A, Kercher J, Sherman S (2011) Clinical cartilage restoration: evolution and overview. Clin Orthop Relat Res 469: 2696-2705.

Fishbein KW, Canuto HC, Bajaj P, Camacho NP, Spencer RG (2007) Optimal methods for the preservation of cartilage samples in MRI and correlative biochemical studies. Magn Reson Med 57: 866-873.

Fontaine P, Blond L, Alexander K, Beauchamp G, Richard H, Laverty S (2013) Computed tomography and magnetic resonance imaging in the study of joint development in the equine pelvic limb. Vet J 197: 103-111.

Frisbie DD, Stewart MC (2011) Cell-based therapies for equine joint disease. Vet Clin North Am Equine Pract 27: $335-349$.

Goodwin DW, Dunn JF (1998) High-resolution magnetic resonance imaging of articular cartilage: correlation with histology and pathology. Top Magn Reson Imaging 9: 337-347.

Grunder W (2006) MRI assessment of cartilage ultrastructure. NMR Biomed 19: 855-876.

Grunder W, Wagner M, Werner A (1998) MRmicroscopic visualization of anisotropic internal cartilage structures using the magic angle technique. Magn Reson Med 39: 376-382.

Grunder W, Kanowski M, Wagner M, Werner A (2000) Visualization of pressure distribution within loaded joint cartilage by application of angle-sensitive NMR microscopy. Magn Reson Med 43: 884-891.

Hayter C, Potter H (2011) Magnetic resonance imaging of cartilage repair techniques. J Knee Surg 24: 225-240.

Heegaard JH, Beaupre GS, Carter DR (1999) Mechanically modulated cartilage growth may regulate joint surface morphogenesis. J Orthop Res 17: 509-517.
Holopainen JT, Brama PA, Halmesmaki E, Harjula T, Tuukkanen J, van Weeren PR, Helminen HJ, Hyttinen MM (2008) Changes in subchondral bone mineral density and collagen matrix organization in growing horses. Bone 43: 1108-1114.

Hughes LC, Archer CW, ap Gwynn I (2005) The ultrastructure of mouse articular cartilage: collagen orientation and implications for tissue functionality. A polarised light and scanning electron microscope study and review. Eur Cell Mater 9: 68-84.

Hunziker EB, Kapfinger E, Geiss J (2007) The structural architecture of adult mammalian articular cartilage evolves by a synchronized process of tissue resorption and neoformation during postnatal development. Osteoarthritis Cartilage 15: 403-413.

Hurtig M, Green S, L., Dobson H, Mikuni-Takagaki Y, Choi J (1993) Correlative study of defective cartilage and bone growth in foals fed a low-copper diet. Equine Vet $\mathrm{J}$ Suppl. 16: 66-73.

Hwang WS, Li B, Jin LH, Ngo K, Schachar NS, Hughes GN (1992) Collagen fibril structure of normal, aging, and osteoarthritic cartilage. J Pathol 167: 425-433.

Hyttinen MM, Holopainen J, Weeren PRv, Firth EC, Helminen HJ, Brama PAJ (2009) Changes in collagen fibril network organization and proteoglycan distribution in equine articular cartilage during maturation and growth. J Anat 215: 584-591.

Jaramillo D, Connolly SA, Mulkern RV, Shapiro F (1998) Developing epiphysis: MR imaging characteristics and histologic correlation in the newborn lamb. Radiology 207: 637-645.

Jaramillo D, Villegas-Medina OL, Doty DK, Rivas R, Strife K, Dwek JR, Mulkern RV, Shapiro F (2004) Agerelated vascular changes in the epiphysis, physis, and metaphysis: normal findings on gadolinium-enhanced MRI of piglets. AJR Am J Roentgenol 182: 353-360.

Jomha NM, Anoop PC, McGann LE (2004) Intramatrix events during cryopreservation of porcine articular cartilage using rapid cooling. J Orthop Res 22: 152-157.

Julkunen P, Korhonen RK, Nissi MJ, Jurvelin JS (2008) Mechanical characterization of articular cartilage by combining magnetic resonance imaging and finite-element analysis: a potential functional imaging technique. Phys Med Biol 53: 2425-2438.

Julkunen P, Harjula T, Iivarinen J, Marjanen J, Seppanen K, Narhi T, Arokoski J, Lammi MJ, Brama PA, Jurvelin JS, Helminen HJ (2009) Biomechanical, biochemical and structural correlations in immature and mature rabbit articular cartilage. Osteoarthritis Cartilage 17: 1628-1638.

Julkunen P, Iivarinen J, Brama PA, Arokoski J, Jurvelin JS, Helminen HJ (2010) Maturation of collagen fibril network structure in tibial and femoral cartilage of rabbits. Osteoarthritis Cartilage 18: 406-415.

Kangarlu A, Gahunia HK (2006) Magnetic resonance imaging characterization of osteochondral defect repair in a goat model at $8 \mathrm{~T}$. Osteoarthritis Cartilage 14: 52-62.

Khan IM, Evans SL, Young RD, Blain EJ, Quantock AJ, Avery N, Archer CW (2011) FGF2 and TGFbeta1 induce precocious maturation of articular cartilage. Arthritis Rheum 63: 3417-3427. 
Kim DJ, Suh JS, Jeong EK, Shin KH, Yang WI (1999) Correlation of laminated MR appearance of articular cartilage with histology, ascertained by artificial landmarks on the cartilage. J Magn Reson Imaging 10: 57-64.

Kim M, Foo LF, Uggen C, Lyman S, Ryaby JT, Moynihan DP, Grande DA, Potter HG, Pleshko N (2010) Evaluation of early osteochondral defect repair in a rabbit model utilizing fourier transform-infrared imaging spectroscopy, magnetic resonance imaging, and quantitative T2 mapping. Tissue Eng Part C Methods 16: 355-364.

Klein TJ, Chaudhry M, Bae WC, Sah RL (2007) Depthdependent biomechanical and biochemical properties of fetal, newborn, and tissue-engineered articular cartilage. J Biomech 40: 182-190.

Landis JR, Koch GG (1977) An application of hierarchical kappa-type statistics in the assessment of majority agreement among multiple observers. Biometrics 33: 363-374.

Laouar L, Fishbein K, McGann LE, Horton WE, Spencer RG, Jomha NM (2007) Cryopreservation of porcine articular cartilage: MRI and biochemical results after different freezing protocols. Cryobiology 54: 36-43.

Laverty S, Ionescu M, Marcoux M, Boure L, Doize B, Poole AR (2000) Alterations in cartilage type-II procollagen and aggrecan contents in synovial fluid in equine osteochondrosis. J Orthop Res 18: 399-405.

Laverty S, Okouneff S, Ionescu M, Reiner A, Pidoux I, Webber C, Rossier Y, Billinghurst RC, Poole AR (2002) Excessive degradation of type II collagen in articular cartilage in equine osteochondrosis. J Orthop Res 20: 1282-1289.

Lecocq M, Girard CA, Fogarty U, Beauchamp G, Richard H, Laverty S (2008) Cartilage matrix changes in the developing epiphysis: early events on the pathway to equine osteochondrosis? Equine Vet J 40: 442-454.

Link TM, Stahl R, Woertler K (2007) Cartilage imaging: motivation, techniques, current and future significance. Eur Radiol 17: 1135-1146.

Mahmoodian R, Leasure J, Philip P, Pleshko N, Capaldi F, Siegler S (2011) Changes in mechanics and composition of human talar cartilage anlagen during fetal development. Osteoarthritis Cartilage 19: 1199-1209.

Menezes NM, Gray ML, Hartke JR, Burstein D (2004) T2 and T1rho MRI in articular cartilage systems. Magn Reson Med 51: 503-509.

Mosher TJ, Dardzinski BJ (2004) Cartilage MRI T2 relaxation time mapping: overview and applications. Semin Musculoskelet Radiol 8: 355-368.

Murray PD, Drachman DB (1969) The role of movement in the development of joints and related structures: the head and neck in the chick embryo. J Embryol Exp Morphol 22: 349-371.

Nieminen MT, Toyras J, Rieppo J, Hakumaki JM, Silvennoinen J, Helminen HJ, Jurvelin JS (2000) Quantitative MR microscopy of enzymatically degraded articular cartilage. Magn Reson Med 43: 676-681.

Nieminen MT, Rieppo J, Toyras J, Hakumaki JM, Silvennoinen J, Hyttinen MM, Helminen HJ, Jurvelin JS (2001) T2 relaxation reveals spatial collagen architecture in articular cartilage: a comparative quantitative MRI and polarized light microscopic study. Magn Reson Med 46: 487-493.

Nissi MJ, Rieppo J, Toyras J, Laasanen MS, Kiviranta I, Jurvelin JS, Nieminen MT (2006) T(2) relaxation time mapping reveals age- and species-related diversity of collagen network architecture in articular cartilage. Osteoarthritis Cartilage 14: 1265-1271.

Olivier P, Loeuille D, Watrin A, Walter F, Etienne S, Netter P, Gillet P, Blum A (2001) Structural evaluation of articular cartilage: potential contribution of magnetic resonance techniques used in clinical practice. Arthritis Rheum 44: 2285-2295.

Olstad K, Ytrehus B, Ekman S, Carlson CS, Dolvik NI (2007) Early lesions of osteochondrosis in the distal tibia of foals. J Orthop Res 25: 1094-1105.

Onyekwelu I, Goldring MB, Hidaka C (2009) Chondrogenesis, joint formation, and articular cartilage regeneration. J Cell Biochem 107: 383-392.

Recht MP, Goodwin DW, Winalski CS, White LM (2005) MRI of articular cartilage: revisiting current status and future directions. AJR Am J Roentgenol 185: 899-914.

Reiter DA, Peacock A, Spencer RG (2011) Effects of frozen storage and sample temperature on water compartmentation and multiexponential transverse relaxation in cartilage. Magn Reson Imaging 29: 561-567.

Responte DJ, Natoli RM, Athanasiou KA (2007) Collagens of articular cartilage: structure, function, and importance in tissue engineering. Crit Rev Biomed Eng 35: 363-411.

Rieppo J, Hyttinen MM, Halmesmaki E, Ruotsalainen H, Vasara A, Kiviranta I, Jurvelin JS, Helminen HJ (2009) Changes in spatial collagen content and collagen network architecture in porcine articular cartilage during growth and maturation. Osteoarthritis Cartilage 17: 448-455.

Riesle J, Hollander AP, Langer R, Freed LE, VunjakNovakovic G (1998) Collagen in tissue-engineered cartilage: types, structure, and crosslinks. J Cell Biochem 71: 313-327.

Sweat F, Puchtler H, Rosenthal SI (1964) Sirius red F3ba as a stain for connective tissue. Arch Pathol 78: 69-72.

Uhl M, Lahm A, Bley TA, Haberstroh J, Mrosek E, Ghanem N, Erggelet C (2005) Experimental autologous osteochondral plug transfer in the treatment of focal chondral defects: magnetic resonance imaging signs of technical success in sheep. Acta Radiol 46: 875-880.

van de Lest CH, Brama PA, van El B, DeGroot J, van Weeren PR (2004) Extracellular matrix changes in early osteochondrotic defects in foals: a key role for collagen? Biochim Biophys Acta 1690: 54-62.

van Turnhout MC, Haazelager MB, Gijsen MA, Schipper H, Kranenbarg S, van Leeuwen JL (2008) Quantitative description of collagen structure in the articular cartilage of the young and adult equine distal metacarpus. Anim Biol 58: 353-370.

van Turnhout MC, Schipper H, Engel B, Buist W, Kranenbarg S, van Leeuwen JL (2010) Postnatal development of collagen structure in ovine articular cartilage. BMC Dev Biol 10: 62.

van Turnhout MC, Kranenbarg S, van Leeuwen JL (2011) Contribution of postnatal collagen reorientation 
to depth-dependent mechanical properties of articular cartilage. Biomech Model Mechanobiol 10: 269-279.

Varich LJ, Laor T, Jaramillo D (2000) Normal maturation of the distal femoral epiphyseal cartilage: agerelated changes at MR imaging. Radiology 214: 705-709.

Verstraete KL, Almqvist F, Verdonk P, Vanderschueren G, Huysse W, Verdonk R, Verbrugge G (2004) Magnetic resonance imaging of cartilage and cartilage repair. Clin Radiol 59: 674-689.

Watanabe A, Boesch C, Anderson SE, Brehm W, Mainil Varlet P (2009) Ability of dGEMRIC and T2 mapping to evaluate cartilage repair after microfracture: a goat study. Osteoarthritis Cartilage 17: 1341-1349.

Williamson AK, Chen AC, Masuda K, Thonar EJ, Sah RL (2003) Tensile mechanical properties of bovine articular cartilage: variations with growth and relationships to collagen network components. J Orthop Res 21: 872-880.

Xia Y (2003) The total volume and the complete thickness of articular cartilage determined by MRI. Osteoarthritis Cartilage 11: 473-474.

Xia Y, Moody JB, Burton-Wurster N, Lust G (2001) Quantitative in situ correlation between microscopic MRI and polarized light microscopy studies of articular cartilage. Osteoarthritis Cartilage 9: 393-406.

Xia Y, Moody JB, Alhadlaq H, Hu J (2003) Imaging the physical and morphological properties of a multi-zone young articular cartilage at microscopic resolution. J Magn Reson Imaging 17: 365-374.

Yazici Z, Kline-Fath BM, Laor T, Tinkle BT (2010) Fetal MR imaging of Kniest dysplasia. Pediatr Radiol 40: 348-352.

\section{Discussion with Reviewers}

Reviewer I: Have the authors thought of using quantitative MR sequences (T2, T1rho mapping or gagCEST imaging) for their study? Maybe these quantitative sequences would have given a greater dynamic range and reproducibility for scoring the cartilage.

Authors: We agree with the reviewer that quantitative sequences could have improved the quality of assessment of cartilage and would have been very interesting to perform. However, when this study was performed we did not have access to quantitative MRI techniques. We are currently continuing this work employing 3T MRI and T2, T1rho mapping, in addition to other novel imaging sequences, to study juvenile cartilage development.

Reviewer II: The samples were frozen at $-20^{\circ} \mathrm{C}$. At this temperature ice crystal formation can occur that may influence the orientation of the collagen fibrils. Can you comment on these methods and how you verified that the cartilage orientations were unaffected?

Authors: The MRI imaging methods were based on similar studies of epiphyseal development in limbs that had been frozen at $-20^{\circ} \mathrm{C}$ (Connolly et al., 2004; Olivier et al., 2001). The ideal experimental design to avoid potential cartilage collagen structural changes in these specimens would be to image and process all the specimens immediately following harvest. It is not usually practical, or possible, to image all limb specimens on the day of harvest and then process them. Joints or limbs are often stored frozen when there is a delay in imaging due to either a delay in collection of all specimens or scheduling issues, to attempt to prevent chondrocyte catabolic enzyme degradation of the cartilage matrix. Nevertheless, this is a trade-off as the freeze-thaw process, particularly when repeated, may lead to ice crystal accumulation and potential disruption of the cartilage collagen structure (ap Gwynn et al., 2000; Jomha et al., 2004). On the other hand, investigators observed that small ice crystals do not affect the collagen orientation in mice articular cartilage samples on scanning electron microscopy examination (Hughes et al., 2005). Only one freeze-thaw cycle was performed on the specimens in the current study as the freeze-thaw, not storage, contributes to the cartilage matrix damage.

The equine foetuses were supplied by an abattoir and were frozen immediately on-site and stored until collection. Ideally, a foetal limb specimen should have been assessed with the selected MRI sequences prior to and post freezing to determine whether the image changed due to potential structural changes induced by ice crystal formation. Histological analysis of the collagen structure could also have been assessed on sections obtained from a femur prior to and post freezing to identify any collagen structural alterations due to freezing. We have not observed a difference in Safranin O staining (for assessment of proteoglycans) pre- and post-freezing in foetal articular cartilage (unpublished data), but have not specifically addressed collagen structure. We will do so in future studies.

Studies on the effects of freezing on cartilage MRI T2 relaxation have been performed principally on cartilage explants. The results of these studies are contradictory but some have suggested that freezing may alter collagen structure (Fishbein et al., 2007; Laouar et al., 2007). Freezing and thawing of bovine nasal cartilage explants resulted in small changes in T2 (Fishbein et al., 2007, Reiter et al., 2011). It is not known if these observations of freezing effects on isolated cartilage explants can be extrapolated to complete intact joints.

Reviewer II: The authors mentioned that the magic angle affects the signal intensity of the MR measurements. Can they discuss how much this effect has affected the MR signal and contrast in their experiments?

Authors: The magic angle effect has the potential ability to alter contrast between cartilage layers by increasing the signal intensity of otherwise hypointense layers. This effect is strongly dependent on the angle of the cartilage structure relative to the static magnetic field. It is therefore unlikely that this effect has precluded identification of cartilage layers diffusely in a rounded joint surface such as the femoral trochlea or condyles. We did not get the impression that this artefact was present while reading the images.

Reviewer II: Could the authors think of other (smaller) animal models, which are suitable to study the maturation of the distal femoral epiphyseal articular surface? Especially when the MRI experiments are carried out at 
high magnetic field strength (7T and above) and customised MR detectors are used.

Authors: Rodent animal models could potentially be suitable for future studies of distal femoral epiphyseal articular surface development employing higher resolution MRI with customised coils as MRI (7T) has been employed to monitor osteoarthritis related changes in rat femurs (Goebel et al., 2010).

\section{Additional Reference}

Goebel JC, Bolbos R, Pham M, Galois L, Rengle A, Loeuille D, Netter P, Gillet P, Beuf O, Watrin-Pinzano A (2010) In vivo high-resolution MRI (7T) of femoro-tibial cartilage changes in the rat anterior cruciate ligament transection model of osteoarthritis: a cross-sectional study. Rheumatology (Oxford) 49: 1654-1664. 